\title{
Association of dietary calcium intake, total and ionized serum calcium levels with preeclampsia in Ethiopia
}

\author{
Rahel D. Gebreyohannes ${ }^{1 *}$, Ahmed Abdella' ${ }^{1}$, Wondimu Ayele ${ }^{2}$ and Ahizechukwu C. Eke ${ }^{3}$
}

\begin{abstract}
Background: Preeclampsia is a well-known cause of maternal mortality and morbidity in Ethiopia. The exact pathophysiology has not been fully understood. Calcium and magnesium deficiencies have been given emphasis to play roles in the pathophysiology. Although evidence is abundant, they are equivocal. The study aimed to see the association of dietary calcium intake, serum total calcium level and ionized calcium level with preeclampsia. It also evaluated the association between dietary calcium intake and serum calcium levels.
\end{abstract}

Materials and methods: An unmatched case-control study was conducted in Gandhi Memorial, Tikur Anbessa, and Zewditu Memorial Hospitals, all in Addis Ababa, between October to December, 2019. Cases were 42 women with preeclampsia and controls were 42 normotensive women. The medical and obstetric history was gathered using a structured questionnaire and the dietary calcium intake information using a 24-h dietary recall. The serum levels of total serum calcium and ionized (free) calcium were measured using an inductively coupled mass spectrophotometer. Bivariate and multivariate logistic regression and Pearson correlation test were utilized during data analysis.

Results: In comparison with controls, women with preeclampsia had lower mean ( \pm 1SD) levels of ionized calcium level (1.1 mmol/I \pm 0.11$)$, total serum calcium level $(1.99 \mathrm{mmol} / \mathrm{I} \pm 0.35)$ and lower median (IQR) dietary calcium intake (704 mg/24 h,458-1183). The odds of having preeclampsia was almost eight times greater in those participants with low serum ionized calcium level $(\mathrm{OR} 7.5,95 \% \mathrm{Cl}$ 2.388-23.608) and three times higher in those with low total serum calcium level (OR 3.0, 95\% Cl 1.024-9.370). Low dietary calcium intake also showed statistically significant association with preeclampsia (OR 3.4, 95\% Cl 1.092 -10.723). Serum ionized calcium level and total serum calcium level showed positive correlation of moderate strength $(p=0.004, r=0.307)$, but no correlation was found between dietary calcium intake with both forms of serum calcium levels.

Conclusion: This study showed significant association between low dietary calcium intake and low serum calcium levels with preeclampsia, hence this can be used as a supportive local evidence for the current context-specific recommendation of calcium supplementation in societies with low-dietary calcium consumption in an attempt to prevent preeclampsia, therefore implementation study should be considered in Ethiopia to look for the feasibility of routine supplementation.

Keywords: Pre-eclampsia, Total serum calcium level, Dietary calcium, lonized serum calcium level

*Correspondence: raheldemissewgy@gmail.com

1 Department of Obstetrics and Gynecology, Addis Ababa University,

College of Health Sciences, Addis Ababa, Ethiopia

Full list of author information is available at the end of the article

\section{Background}

High blood pressure (with or without proteinuria) is a major cause of maternal and perinatal morbidity and mortality worldwide [1, 2]. Preeclampsia complicates 
about $5-10 \%$ of all pregnancies and accounts for $>50,000$ maternal deaths each year $[2,3]$. In studies done in different parts of Ethiopia including the capital Addis Ababa, prevalence ranged between $7.2 \%$ and $8.4 \%$ [4-8].

The etiology of this condition remains unknown, although believed to be multifactorial. Calcium deficiency is among the etiologies that have been implicated [9]. Currently, there is an increased interest in the management and prevention of preeclampsia with nutritional supplements, such as the use of calcium and magnesium supplementation. However, reports from clinical studies are inconsistent on the role of calcium and magnesium in the etiology and prevention of preeclampsia. Reports from different countries such as India, Sudan, South Korea, and Iran have shown significant relationships between low serum calcium levels and preeclampsia [1013], whereas, other studies from Ghana, Nigeria, South Africa and India have shown no difference in serum calcium levels in hypertensive and normotensive pregnant women [14-16].

The decrease in dietary calcium intake will decrease serum calcium concentrations, thereby inducing the release of parathyroid hormone, which in-turn increases the intracellular calcium levels, causing vasoconstriction [17]. But there are several evidences that the serum level of calcium does not solely depend on dietary intake of calcium. A study in India reported the association of dietary calcium intake, serum calcium level and preeclampsia, and noted that study subjects had normal serum calcium level despite very low intake of dietary calcium during pregnancy. The possible explanation for their finding was the possibility of greater exposure of the study participants to sunlight and increased vitamin D production. This may be possible as the serum calcium levels are tightly regulated by hormones (Parathyroid hormone and Calcitonin) and the prohormone Vitamin D and through organs (bone, kidney and small intestine) through complex feedback loops $[17,18]$.

About half of the plasma calcium is found in ionized (free) form and the rest is bound to protein (40\%) and anions. The ionized calcium is the fraction that is biologically active and tightly regulated by hormones. During pregnancy, the ionized calcium remains constant throughout gestation, but the total calcium concentration varies significantly due to hemodilution [18]. It is therefore evident that serum ionized calcium is by far the most important index to know the actual calcium status of the body compared to the total serum calcium level.

World Health Organization (WHO) currently recommends calcium supplementation at a dose of $1.5-2 \mathrm{~g} /$ day starting in the first half of pregnancy for prevention of preeclampsia for those populations with generally low dietary calcium intake. However, the recommendation for calcium supplementation is still context specific because the implementation clearly may not be easy considering the cost and burden of the drugs to incorporate the recommendation in to the local guideline [19].

Typically, daily calcium intake in low-income countries is $300-600 \mathrm{mg} /$ day compared with $855 \mathrm{mg} /$ day in the United Kingdom and $969 \mathrm{mg} /$ day in France [20]. A big National Survey has been done to assess the dietary calcium intake of the average Ethiopian women in the child bearing age, and demonstrated that dietary calcium intake of women in this age group was very low (i.e. $317.32 \mathrm{mg} /$ day) compared with the recommended daily allowance (RDA) [21]. The RDA should be at least $1,000 \mathrm{mg} /$ day for pregnant women. With all the evidences of low dietary calcium intake and low serum calcium levels in the Ethiopian community, no study has been done to demonstrate any associations between serum calcium concentrations (both total and ionized) and dietary calcium intake and the development of pre-eclampsia in Ethiopian women, prompting the research gap filled by this study. Hence, this study aimed to see the association of dietary calcium intake, serum total calcium level and ionized calcium level with preeclampsia. It also evaluated the correlation between dietary calcium intake and serum calcium levels and the correlation between serum total calcium level and ionized calcium level.

\section{Methods}

Study design

Unmatched case-control study was done at three big hospitals in the capital Addis Ababa: Gandhi Memorial Hospital, Tikur Anbessa Hospital and Zewditu Memorial Hospital. The study was completed in 84 participants (42 cases and 42 controls). The required sample size was determined using unmatched case control study sample size calculation formula. Given the lack of concrete information from previous studies on the prevalence of low dietary calcium intake and low serum calcium level among preeclamptic women in similar setting, in order to get representative sample size, we assumed hypothetically identified odds ratio of 4.0 that could yield the representative sample size, a confidence level of $95 \%$ and power of $80 \%$. Adjustment was made for population size (less than 10 times from estimated sample size) using finite population correction factor.

\section{Data collection procedures}

Cases were recruited based on the current (2019) International Society for the Study of Hypertension in Pregnancy (ISSHP) definition of preeclampsia which is, systolic blood pressure at $\geq 140 \mathrm{mmHg}$ and/or diastolic blood pressure at $\geq 90 \mathrm{mmHg}$ on at least two occasions measured 4 hours apart in previously normotensive 
women and is accompanied by one or more of the following new onset conditions at or after 20 weeks of gestation: proteinuria (i.e. $\geq 30 \mathrm{mg} / \mathrm{mol}$ protein: creatinine ratio; $\geq 300 \mathrm{mg} / 24 \mathrm{~h}$; or $\geq 2+$ dipstick); Evidence of other maternal organ dysfunction, including: acute kidney injury (creatinine $\geq 90 \mu \mathrm{mol} / \mathrm{L} ; 1 \mathrm{mg} / \mathrm{dL}$ ); liver involvement (elevated transaminases, e.g. alanine aminotransferase or aspartate aminotransferase $>40 \mathrm{IU} / \mathrm{L}$ ) with or without right upper quadrant or epigastric abdominal pain; neurological complications (e.g. eclampsia, altered mental status, blindness, stroke, clonus, severe headaches, and persistent visual scotomata); or hematological complications (thrombocytopenia-platelet count $<150$ $000 / \mu \mathrm{l}$, disseminated intravascular coagulation, hemolysis); or uteroplacental dysfunction (such as fetal growth restriction, abnormal umbilical artery doppler waveform analysis, or stillbirth) [22]. Cases were selected from the antenatal clinics (ANC), but sometimes followed to the antepartum ward for repeat blood pressure (BP) measurement. Incident cases were chosen to decrease recall bias.

Inclusion criteria for the cases were women aged $\geq 18$ years, gestational age $\geq 20$ weeks and singleton pregnancy. Those with chronic hypertension, women on anti-hypertensive and anti-convulsant treatment, diabetes mellitus, renal disease, known thyroid and adrenal disease, intrauterine fetal death and those taking antenatal vitamin and mineral supplementation were excluded.

Controls were selected from the hospitals too (source population). Recruiting neighborhood and population controls was not possible in our setting, as the likelihood of finding a healthy non-hypertensive pregnant woman in the neighborhood was unlikely. Any risk factors that could contribute for preeclampsia in the current or previous pregnancies, such as antepartum hemorrhage (APH) and twin pregnancies, were excluded. Controls were women who came for normal ANC follow up, or with obstetric conditions like previous cesarean section scar, or women that could otherwise follow pregnancy at the health centers.

Systematic random sampling technique was used to recruit participants. With the assumption that there could be around 250 cases of preeclampsia in the data collection period, we recruited every sixth patient with preeclampsia and a corresponding number of controls was selected.

Recruitment of cases and controls and coding were done by the principal investigator and the treating physician at ANC and emergency room. Data collectors, who were nurses with Bachelor of Science (BSC) degree, were masked before they took the dietary recall, then unmasked after completing the collection of data related to the dietary recall, as information from patient cards was mandatory to complete the questionnaire. The diagnosis of preeclampsia was made by the consultation physician, and evidences were carefully recorded. Gestational age was determined with the most accurate obstetric estimate, using menstrual history, symphysisfundal height at the initial antenatal (booking) visit or ultrasound scan, when available. The questionnaire comprised demographic details, booking details of the index pregnancy, general medical and health details, a dietary questionnaire. Blood pressure was measured by trained personnel using a manual pneumatic mercury sphygmomanometer, and utilizing the fifth Korotkoff sound. A repeat measurement was recorded after $4 \mathrm{~h}$ to ensure consistency in blood pressure measurement.

Ten milliliters of blood from antecubital fossa was taken, centrifuged, and refrigerated at 2-8 degree centigrade until analyzed. Total serum calcium and ionized serum calcium were determined by using an inductively coupled plasma mass spectrometer. Twenty-four-hour dietary recall was done and calcium content in the meals of the participants were calculated using the food composition table for use in Ethiopia, Part III, developed in 1995-1997 [23].

Data was collected from October 3 to December 25, 2019.

\section{Data analysis}

Data was analyzed using SPSS version 25 (IBM, Armonk, NY, USA). Descriptive analyses, Chi-square test, univariate and multivariate logistic regressions were done. Odds ratios (OR), adjusted OR and 95\% confidence intervals (CI) were calculated. Pearson/Spearman correlation was performed. $P$ value of less than 0.05 was considered statistically significant. We used t-test statistic to see the association of the variables age, parity, gestational age and body mass index with preeclampsia. The association between dietary calcium intake and total and ionized serum calcium levels, educational level, body mass index, parity, age group and gestational age, income was seen using Chi-square test. Bivariate logistic regression was used to measure the relationship of each covariate with the outcome variable (preeclampsia). Variables that were associated with the outcome variable with $p$-value of $<0.2$ in the univariate analysis and clinically relevant variables that were known to associate with preeclampsia despite their $p$-values in the univariate analysis were included in to the multiple logistic regression model. Hence, the variables imputed in the multiple logistic regression model were parity, gestational age, total serum calcium level, ionized calcium level and dietary calcium amount. Pearson correlation was used to see if dietary calcium intake, total serum calcium level and ionized calcium level correlated with each other. 
Reference (normal) values taken were $2.1-2.55 \mathrm{mmol} / \mathrm{l}$ for total serum calcium level, $1.16-1.32 \mathrm{mmol} / \mathrm{l}$ for ionized calcium level and Recommended Dietary Allowance (RDA) of $\geq 1000 \mathrm{mg} / 24 \mathrm{~h}$ for dietary calcium intake.

\section{Results}

Majority of the cases $(n=33,78.6 \%)$, had preeclampsia with severe features. The remaining 9 cases $(21.4 \%)$ had preeclampsia without severity features. Severe hypertension $(\mathrm{n}=25,60 \%)$ and other severity symptoms $(\mathrm{n}=26$, $62 \%$ ) of preeclampsia were equally common. Six patients (18.2\%) had HELLP syndrome (hemolysis, elevated liver enzymes, low platelets). Two of the cases (6\%) had acute kidney injury, four (9.5\%) had intrauterine growth restriction (IUGR), and one had eclampsia.

Most of the controls $(n=14,57.1 \%)$ had low risk pregnancies, while the remaining controls had scarred uterus $(\mathrm{n}=11,26.2 \%)$, post-term pregnancies $(\mathrm{n}=4,9.5 \%)$, malpresentations $(\mathrm{n}=2,4.8 \%)$, and premature rupture of membranes (PROM) $(\mathrm{n}=1,2.4 \%)$.

Nineteen percent $(\mathrm{n}=8)$ of the cases and $38.1 \%$ $(\mathrm{n}=16)$ of the controls had gone to the university. Majority $(n=24,57.1 \%)$ of the controls and $40.4 \%(n=17)$ of cases had gone to secondary school or beyond, the illiteracy rate of the women in both groups was low. The median per capita income of the cases was 411 USD (IQR 308-616) and the controls' was 616 USD (IQR 411-821). Both income medians were below the GDP (2019) per capita income of Ethiopia [24]. Regarding employment, $52.4 \%(n=22)$ of the controls and $59.5 \%(n=25)$ of the cases were unemployed.

Both groups were comparable in their obstetric characteristics (age, parity, body mass index/ BMI) except the mean gestational age (GA) of the controls $($ mean $\pm 1 S D=38.50 \pm 3.64)$ was significantly higher $(p=0.04)$ than that of the cases (mean $\pm 1 \mathrm{SD}=36.68 \pm 3.83$ ) (Table 1$)$.

The mean $( \pm 1 \mathrm{SD})$ total serum calcium level and ionized calcium level in the preeclamptic women was found

Table 1 Comparison of the means (sd) of obstetric characteristics of the case and control groups

\begin{tabular}{|c|c|c|c|c|c|}
\hline \multirow[t]{2}{*}{ Variable } & \multicolumn{2}{|c|}{ Cases $(N=42)$} & \multicolumn{2}{|c|}{$\begin{array}{l}\text { Controls } \\
(N=42)\end{array}$} & \multirow[t]{2}{*}{$P$} \\
\hline & Mean & $S D$ & Mean & $S D$ & \\
\hline Age (years) & 27 & 6 & 27 & 4 & 0.824 \\
\hline GA (weeks) & 36.68 & 3.83 & 38.50 & 3.64 & 0.04 \\
\hline Parity & 0.73 & 1.05 & 1.04 & 1.68 & 0.083 \\
\hline Pre-pregnancy BMI $\left(\mathrm{kg} / \mathrm{m}^{2}\right)$ & 23.29 & 3.25 & 23.22 & 4.06 & 0.63 \\
\hline Pregnancy BMI $\left(\mathrm{Kg} / \mathrm{m}^{2}\right)$ & 26.20 & 3.47 & 26.15 & 4.21 & 0.897 \\
\hline
\end{tabular}

GA Gestational age, BMI Body mass index, SD Standard deviation, $N$ Number to be in the hypocalcemia range-lower than the serum calcium concentration in the control groups. Twentynine (nearly $70 \%$ ) of the cases and $n=15(35.7 \%$ ) of the controls had low total serum calcium concentrations. Twenty-five (around 60\%) controls had normal level of total serum calcium level whereas, only $n=11(26 \%)$ of the women with preeclampsia had their total calcium level in the normal range (Table 2).

Majority of the cases $(n=29,69 \%)$ were found to have low ionized calcium level, whereas 9 of the controls (21.4\%) had serum ionized calcium level below the reference range.

The median dietary calcium intake in the cases was lower than those in the controls and the normal range. Almost half of the controls $(n=24,51 \%)$ had a dietary calcium intake equal to or more than the RDA, whereas only one third ( $\mathrm{n}=15,35 \%)$ of the cases had an adequate dietary calcium intake (Table 2).

Univariate logistic regression showed that serum ionized calcium level and total serum calcium level showed statistically significant association with preeclampsia. Having low levels of both types of serum calcium levels is a risk for preeclampsia. Similarly, having the recommended dietary calcium intake was to the direction of protection from preeclampsia, even though statistically not significant. These associations also persisted and dietary calcium intake became statistically significant when imputed in the multivariate analysis (Table 3).

When compared to pregnancies which were $\geq 37$ weeks, having a preterm pregnancy increased the risk of preeclampsia on univariate logistic regression, but when gestational age was imputed into the multiple logistic regression model, it lost its statistical significance (Table 3).

On multivariate analysis, the odds of having preeclampsia was almost eight times higher among those participants with low ionized calcium level (OR 7.5, 95\% CI 2.388-23.608) and three times higher in those with low total serum calcium level (OR 3.0, 95\% CI 1.024-9.370). Low dietary calcium intake also showed statistically significant association with preeclampsia (OR 3.4, CI 1.092 -10.723). (Table 3) Age, parity, BMI, education, employment and income did not show any association with preeclampsia on univariate logistic regression.

On Pearson correlation, Serum ionized calcium level and total serum calcium level showed a positive correlation of moderate strength $(p=0.004, r=0.307)$. But dietary calcium intake was not found to correlate with both total serum calcium level and ionized calcium level (Table 4).

Chi square test did not show significant association between dietary calcium intake and educational level, employment, income, BMI, parity, age group, gestational age, total and ionized serum calcium levels. 
Table 2 Comparison of the means/medians of dietary calcium intake, total serum calcium level and ionized calcium levels in case and control groups

\begin{tabular}{llll}
\hline Variable & $\begin{array}{l}\text { Cases } \\
(\mathbf{n}=\mathbf{4 2})\end{array}$ & $\begin{array}{l}\text { Controls } \\
(\mathbf{n}=\mathbf{4 2})\end{array}$ \\
\hline Total serum calcium level (mmol/l), (mean \pm SD) & $1.99 \pm 0.35$ & $2.19 \pm 0.22$ & $\mathbf{P}$ \\
lonized calcium level (mmol/l), (mean \pm SD) & $1.1 \pm 0.11$ & $1.2 \pm 0.13$ & 0.007 \\
Dietary calcium (gram/24 h), (median, lQR) & $704,458-1183$ & $1115,753-1878$ & 0.000 \\
\hline
\end{tabular}

SD Standard deviation, IQR Interquartile range, $n$ Number

Severity of preeclampsia showed statistically significant association with total serum calcium level (OR 0.096, 95\% CI 0.018-0.518), but not with ionized calcium level and dietary calcium intake.

\section{Discussion}

In this study, the mean total serum calcium level in preeclamptic women was lower than the normal reference range and from the mean in the controls. This is in agreement with other studies done in Sudan, India, South-Korea, Japan, Iran and in other low, middle- and high-income countries [10-13]. The level of mean iodized calcium was also low in those women with preeclampsia. During pregnancy, the ionized calcium remains constant throughout gestation unlike the total calcium which is affected by hemodilution of pregnancy and disorders like malnutrition, nephrotic syndrome and liver diseases, hence making ionized calcium level by far the most important index to know the actual calcium status of the body [18].
This study offers an original contribution to the knowledge of possible relationship of ionized calcium level with preeclampsia.

The metabolism of calcium is complicated and its homeostasis depends on hormones, complex feedback loop and dietary calcium intake. But conflicting results are found with regard to the correlation of dietary calcium amount with serum levels of total and ionized calcium, as diet is not the only factor responsible for the serum levels. Our study showed no correlation between dietary calcium intake with either total serum calcium concentrations or ionized serum calcium level on Pearson correlation, but serum ionized calcium level and serum total calcium levels correlated positively $P=0.004$. This lack of correlation of dietary calcium intake and total serum calcium level has also been demonstrated in a study in pregnant women in India and in the study done by Byrne FN et al. in non-pregnant population [17, 25]. Of note, ionized serum calcium concentrations were not analyzed, therefore it was difficult to compare.

Table 3 Univariate and multivariate logistic regression for the factors associated with preeclampsia

\begin{tabular}{|c|c|c|c|c|c|c|}
\hline Variable & Cases & Controls & $p$-value & cOR $(95 \%$ C.I. $)$ & $p$-value & aOR (95\% C.I.) \\
\hline \multicolumn{7}{|l|}{ Parity } \\
\hline Nulliparous & 22 & 17 & 0.28 & $1.62(0.68-3.84)$ & 0.95 & $1.04(0.33-3.27)$ \\
\hline Multiparous & 20 & 25 & Ref & & & \\
\hline \multicolumn{7}{|c|}{ Gestational age (weeks) } \\
\hline$<28$ & 1 & 1 & 0.76 & $1.55(0.09-26.01)$ & 0.10 & $13.60(0.63-294.62)$ \\
\hline $28-34.9$ & 9 & 3 & 0.03 & $4.64(1.13-19.04)$ & 0.13 & $3.69(0.70-19.65)$ \\
\hline $35-36.9$ & 10 & 4 & 0.04 & $3.86(1.08-13.86)$ & 0.12 & $3.52(0.72-17.30)$ \\
\hline$\geq 37$ & 22 & 34 & Ref & & & \\
\hline \multicolumn{7}{|c|}{ lonized Calcium level (mmol/l) } \\
\hline$<1.16$ & 29 & 9 & 0.00 & $8.1(3.05-21.91)$ & 0.00 & $7.51(2.39-23.60)$ \\
\hline$\geq 1.16$ & 13 & 33 & Ref & & & \\
\hline \multicolumn{7}{|c|}{ Total serum calcium level (mmol/l) } \\
\hline$<2.1$ & 29 & 15 & 0.00 & $4.02(1.62-9.97)$ & 0.045 & $3.10(1.02-9.37)$ \\
\hline$\geq 2.1$ & 13 & 27 & Ref & & & \\
\hline \multicolumn{7}{|c|}{ Dietary calcium intake (gm/24 h) } \\
\hline$<1000$ & 27 & 18 & 0.05 & $2.40(1.00-5.78)$ & 0.03 & $3.42(1.10-10.72)$ \\
\hline$\geq 1000$ & 15 & 24 & Ref & & & \\
\hline
\end{tabular}


Table 4 Correlation between total serum calcium, ionized calcium levels and dietary calcium intake

\begin{tabular}{|c|c|c|c|c|}
\hline \multirow[t]{2}{*}{ Variable } & \multicolumn{2}{|c|}{$\begin{array}{l}\text { lonized calcium } \\
\text { level, } \mathrm{mmol} / \mathrm{I}\end{array}$} & \multicolumn{2}{|c|}{$\begin{array}{l}\text { Dietary } \\
\text { calcium } \\
\text { amount, gm/ } \\
\text { day }\end{array}$} \\
\hline & $P$ & $r$ & $P$ & $r$ \\
\hline Total serum calcium level, mmol/l & 0.004 & 0.307 & 0.352 & 0.103 \\
\hline Dietary calcium amount, gm/day & 0.521 & 0.071 & & \\
\hline
\end{tabular}

$P P$-value, $r$ correlation coefficient

In our study, the median dietary calcium intake assessed with 24-hour dietary recall was $704 \mathrm{mg} / 24 \mathrm{~h}$ (IQR 458-1183) among the pre-eclamptic women, and $1115 \mathrm{mg} / 24 \mathrm{~h}$ (IQR 753-1878) in the controls. The calcium intake in the preeclamptic women was lesser than RDA, which at least, is $1000 \mathrm{mg} /$ day during pregnancy. There is no study to compare this result with other findings, but our data shows a better dietary calcium intake in pregnant women in general compared with the general population according to the national survey result, which estimated it to be $317.32 \mathrm{mg} /$ day in women of child bearing age [26].

The multivariate logistic regression showed that low dietary calcium intake was found more in the preeclamptic women compared with the controls (OR 3.4, CI $1.092-10.723)$. There are only few studies done to see whether low dietary calcium intake would be associated with preeclampsia observing conflicting results. A study in India showed association between low dietary calcium intake and preeclampsia and a study done in Canada did not [17].

On multivariate analysis, the odds of having preeclampsia was almost eight times higher among those participants with low ionized calcium level (OR 7.5, 95\% CI 2.388-23.608) and three times higher in those with low total serum calcium level (OR 3.0, 95\% CI 1.024-9.370). This association of low total serum calcium level with preeclampsia has been demonstrated in studies done in India, Sudan, South- Korea and Iran [10-13]. As has been discussed earlier, total calcium concentration is unreliable in predicting the calcium status of the body. In an attempt to evaluate a more reliable measure of body calcium, a study done in South Africa measured hair calcium level in patients with preeclampsia and normotensive women, and found no difference in hair calcium levels between the two groups [16]. We have not found any other study that tried to see the level of ionized calcium level with regard to its relation with preeclampsia to help us compare with our findings.
In this study, when total serum calcium level was normal, the odds of having severe preeclampsia was $91 \%$ less likely compared with those having a low total serum calcium level. This observation of low total serum calcium level was more evident in women with severe forms of preeclampsia, and was reported in a case-control study done in Bangalore, India [27].

The current study has strengths and limitations. This study is probably the first study in English language that observed the relation of preeclampsia with serum ionized calcium level, even though this fact made comparison of the current study with other studies difficult. Although possible efforts were made to reduce the risk of investigator administered bias by masking the data collectors and recall bias by recruiting only incident cases, both types of bias cannot be eliminated completely.

\section{Conclusion}

This study showed significant association between low dietary calcium intake and low serum calcium levels with preeclampsia. Hence, this can be used as a supportive local evidence for the current context-specific recommendation of calcium supplementation in societies with low-dietary calcium consumption in an attempt to prevent preeclampsia. Therefore, implementation study should be considered in Ethiopia to look for the feasibility of the routine supplementation of calcium starting early during pregnancy. Until then, we recommend health education on the importance of adequate dietary consumption of calcium rich foods during pregnancy. Further large-scale study may be warranted to explain the complex interconnection between ionized calcium level and total serum calcium level with preeclampsia.

\section{Supplementary Information}

The online version contains supplementary material available at https://doi. org/10.1186/s12884-021-04005-y.

Additional file 1.

Additional file 2.

Acknowledgements

This article was financially supported by an NIH funded project, Medical Education Partnership Initiative-Junior Faculty (D43TW010143), therefore we acknowledge the funders and the program.

\section{Authors' contributions}

Conceived and designed the study: RD, AA, AE. Performed the study: RD, AA AE. Analyzed the data: RD, WA. Wrote the paper: RD, AA, AE. Provided expertise and editing: $A E, A A, W A$. All the authors provided constructive comments and approved the final version of the manuscript.

\section{Funding}

This study was financially supported by an NIH funded project, Medical Education Partnership Initiative-Junior Faculty (D43TW010143). 


\section{Availability of data and materials}

The datasets used and analyzed during the current study are available from the corresponding author on reasonable request.

\section{Declarations}

\section{Ethics approval and consent to participate}

Ethical clearance was found from the Institutional Review Board (IRB) of Addis Ababa University, College of Health Sciences. Detailed participant information sheet was provided and written informed consent was obtained. Laboratory calcium results were communicated to the participants and their managing physicians, and attached to their medical record. In cases of abnormal serum calcium results, PI made sure participants got appropriate care and treatment. Blood samples were discarded after the completion of data collection as per the standard guideline used by the laboratory.All the experiment protocol for involving humans was in accordance to guidelines of national/international/ institutional or Declaration of Helsinki.

\section{Consent for publication}

Consent for publication was obtained from participants of the study.

\section{Competing interests}

The authors have declared no conflict of interest.

\section{Author details}

'Department of Obstetrics and Gynecology, Addis Ababa University, College of Health Sciences, Addis Ababa, Ethiopia. ${ }^{2}$ Department of Preventive Medicine, Addis Ababa University, School of Public Health, Addis Ababa, Ethiopia. ${ }^{3}$ Division of Maternal Fetal Medicine, Department of Gynecology and Obstetrics, Johns Hopkins University School of Medicine, Baltimore, MD, USA.

Received: 11 January 2021 Accepted: 2 July 2021

Published online: 27 July 2021

\section{References}

1. World Health Organization. Calcium supplementation during pregnancy for prevention of pre-eclampsia and its complications. [www.who.int]. 2018 (https://www.who.int/nutrition/publications/guidelines/calciumsupplementation-recommendation-2018/en/). Accessed 3 Oct 2020.

2. Poon LC, Shennan A, Hyett JA, Kapur A, et al. The International Federation of Gynecology and Obstetrics (FIGO) initiative on pre-eclampsia: a pragmatic guide for first-trimester screening and prevention. Int J Gynecol Obstet. 2019;145:1-33.

3. Hofmeyr G, Lawrie TA, Atallah ÁN, Torloni M. Calcium supplementation during pregnancy for preventing hypertensive disorders and related problems. Cochrane Database Syst Rev. 2018;10:CD001059.

4. Abdella A. Maternal Mortality Trend in Ethiopia. Ethiop J Health Dev. 2010;24:115-22

5. Shegaze M, Markos Y, Estifanos W, Taye I, Gemeda E, et al. Magnitude and associated factors of preeclampsia among pregnant women who attend antenatal care service in public health institutions in Arba Minch Town, Southern Ethiopia. Gynecol Obstet (Sunnyvale). 2016;6:419.

6. Wagnew M, Dessalegn M, Worku A, Nyagero J. Trends of preeclampsia/ eclampsia and maternal and neonatal outcomes among women delivering in Addis Ababa. Pan Afr Med J. 2016:25:12.

7. Seyom E, Abera M, Tesfaye M, Fentahun N. Maternal and fetal outcome of pregnancy related hypertension in Mettu Karl Referral Hospital. Ethiop J Ovarian Res. 2015;8:10.

8. Tessema GA, Tekeste A, Ayele TA. Preeclampsia and associated factors among pregnant women attending antenatal care in Dessie referral hospital, Northeast Ethiopia: a hospital-based study. BMC Pregnancy Childbirth. 2015:15:73

9. Chhabra S, Singh A. Role of calcium in hypertensive disorders of pregnancy current status of research a minireview. J Nutr Disorders Ther. $2017 ; 7: 212$
10. Kanagal DV, Rajesh A, Rao K, Devi UH, Shetty H, Kumari S, Shetty PK. Levels of serum calcium and magnesium in pre-eclamptic and normal pregnancy: a study from coastal India. J Clin Diagn Res. 2014;8(7):OC01-4.

11. Elmugabil A, Hamdan HZ, Elsheikh AE, Rayis DA, Adam I, Gasim Gl. Serum calcium, magnesium, zinc and copper levels in sudanese women with preeclampsia. PLoS ONE. 2016;11(12):e0167495.

12. Kim J, Kim YJ, Lee R, Moon JH, Jo I. Serum levels of zinc, calcium, and iron are associated with the risk of preeclampsia in pregnant women. Nutr Res. 2012:32(10):764-9.

13. Farzin L, Sajadi F. Comparison of serum trace element levels in patients with or without pre-eclampsia. J Res Med Sci. 2012;17(10):938-41.

14. Ephraim RKD, Osakunor DNM, Denkyira SW, Eshun H, Amoah S, Anto EO. Serum calcium and magnesium levels in women presenting with pre-eclampsia and pregnancy-induced hypertension: a case-control study in the Cape Coast metropolis, Ghana. BMC Pregnancy Childbirth. 2014;14:390.

15. Ugwuja El, Famurewa AC, Ikaraoha Cl. Comparison of serum calcium and magnesium between preeclamptic and normotensive pregnant Nigerian women in Abakaliki. Nigeria Ann Med Health Sci Res. 2016;6(1):33-7.

16. Richards DGD, Lindow SW, Carrara H, Knight R, Haswell SJ, Van der Spuy ZM. A comparison of maternal calcium and magnesium levels in preeclamptic and normotensive pregnancies: an observational case-control study. BJOG. 2014;121:327-36.

17. Gupta A, Kant S, Pandav CS, Gupta SK, Rai SK, Misra P. Dietary Calcium intake, serum calcium level, and their association with preeclampsia in rural North India. Indian J Commun Med. 2016;41(3):223-7.

18. Almaghamsi $A$, Almalki MH, Buhary BM. Hypocalcemia in pregnancy: a clinical review update. Oman Med J. 2018;33(6):453-62.

19. World Health Organization. WHO recommendations for prevention and treatment of preeclampsia and eclampsia [wWw.who.int]. 2011 www. who.int/reproductivehealth/publications/maternal_perinatal_health/ 9789241548335/en/index.html. Accessed 2 Oct 2020.

20. Hofmeyr GJ, Belizán JM, von Dadelszen P, Calcium and Pre-eclampsia (CAP) Study Group. Low-dose calcium supplementation for preventing pre-eclampsia: a systematic review and commentary. BJOG. 2014;121(8):951-7.

21. Tesfaye B, Wuehler S, Moges T, Samuel A, Kebede A, Zerfu D, Abera A, Mengistu G, Wodajo B, Birks KA, Jalal C, Kebede A. Making a case for calcium supplementation for prevention of pregnancy hypertension in Ethiopia. Eur J Nutr Food Safety. 2015;5(5):1187-8.

22. Brown MA, Mageec LA, Kennyd LC, Karumanchie SA, McCarthyf FP, Saitog S, Hallh DR, Warreni CE, Adoyij G, Ishaku S. The hypertensive disorders of pregnancy: ISSHP classification, diagnosis \& management recommendations for international practice. Pregnancy Hypertension. 2018;72(1):24-43

23. EHNRI. Food composition table for use in Ethiopia Part III. Addis Ababa: Ethiopian Health and Nutrition Research Institute; 1997.

24. World Bank national accounts data, and OECD National Accounts data files. GDP per capita (current US\$) - Ethiopia. 2019 https://data.world bank.org/indicator/NY.GDP.PCAP.CD?locations=ET

25. Byrne FN, Kinsella S, Murnaghan DJ, Kiely M, Eustace JA. Lack of correlation between calcium intake and serum calcium levels in stable haemodialysis subjects. Nephron Clin Pract. 2009;113(3):c162-8.

26. Ethiopian National Food Consumption survey, Ethiopian Public Health Institute, Addis Ababa Ethiopia, 2013. www.ephi.gov.et

27. Pairu J, Triveni GS, Manohar A. The study of serum calcium and serum magnesium in pregnancy induced hypertension and normal pregnancy. Int J Reprod Contracept Obstet Gynecol. 2015;4(1):30-4.

\section{Publisher's Note}

Springer Nature remains neutral with regard to jurisdictional claims in published maps and institutional affiliations. 